\title{
CONDITIONS OF SAMPLE PREPARATION FOR QUANTITATIVE X-RAY DIFFRACTION OF CEMENT CLINKER
}

\author{
Makio YAMASHITA ${ }^{* 1}$, Yasuo KOGA ${ }^{* 1}$, Hisanobu TANAKA ${ }^{* 1}$ and Yoichiro NAKANISHI ${ }^{* 1}$ \\ *1 UBE-MITSUBISHI CEMENT RESEARCH INSTITUTE, Saitama Center, Cement Group \\ (2270, Yokoze, Yokoze-machi, Chichibu-gun, Saitama 368-8504, Japan)
}

\begin{abstract}
Recent developments in detector performance for powder X-ray diffraction have led to a drastic increase in the speed of measurement. In addition, software development of the Rietveld method has enabled a rapid quantitative analysis of crystalline phases that is expected to be applicable to inplant processes and quality control. However, there has not been sufficient study of how to prepare samples for the measurement of clinker and cement, and how to appropriately grind samples for measurement. This study examines the preparation conditions of clinker and cement samples for fast X-ray diffraction and the Rietveld method. The following conclusions were reached. (1) The fineness of the clinker sample affects the calculated mineral composition, especially for alite and belite. (2) Clinker minerals can be appropriately determined when the Blaine surface area of the specimen is controlled within a range of 3000 to $4500 \mathrm{~cm}^{2} / \mathrm{g}$. (3) The mineral composition determined by the Rietveld method is little affected by difference in the grindability of clinker prepared under the same grinding conditions.

(4) There is no need to grind samples for portland cement measurement.
\end{abstract}

KEY WORDS : X-ray diffraction, Rietveld method, Clinker minerals, Sample preparation, Blaine specific surface

\section{INTRODUCTION}

The cement industry has successfully numerous waste and industrial by-products through stable production facilities and manufacturing technologies. Recently, more than 30 kinds of waste and by-products from other industries have been used as alternative raw materials or thermal energy sources. Such alternative materials and fuels often have different chemical and physical properties than the conventional ones used for cement manufacturing. Therefore expanding using such alternatives in cement manufacture can cause variability of chemical and mineral composition of cement.

A substantial amount of effort is put into processing in the cement industry, such as minimizing the variability of the raw materials and controlling the burning process to ensure uniform quality and optimum cement properties. It is important to control chemical and mineral composition for these influence the properties of the clinker and ultimately of the cement and concrete themselves. ${ }^{1)} \mathrm{X}$-ray fluoresce analysis of chemical composition accomplished online supplies fast and accurate chemical analyses at the cement plant. By using this technology we can reduce the variability of the chemical composition of clinker and cement. The proportion of a given mineral composing clinker is determined by general practice using a calculation, such as the Bogue calculation. However, the result will differ from the actual value, since this method is affected by the amount of minor constituents and the burning conditions of the clinker. Also, the point-count method using a polarization microscope requires considerable time and substantial skill for observation.

The Rietveld method using X-ray diffraction patterns has drawn wide attention because it quantifies the amount and crystallographic characteristics of the phases present in the sample. Recent developments in fast X-ray diffractometers and the evolution of a reliable Rietveld program have reduced the acquisition time to less than 10minutes, attracting the interest of many involved in X-ray diffraction in cement plants. ${ }^{2.3)}$ In sample preparation, only pressed pellets can be used for the powder X-ray diffraction method. Pressed pellets are the first choice for online analysis, 
Table 1 (a) Mineral composition of clinkers

\begin{tabular}{c|c|c|c|c|c}
\hline \multirow{2}{*}{ Clinker } & \multicolumn{5}{|c}{ Mineral composition } \\
\cline { 3 - 6 } & & $\mathrm{C}_{3} \mathrm{~S}$ & $\mathrm{C}_{2} \mathrm{~S}$ & $\mathrm{C}_{3} \mathrm{~A}$ & $\mathrm{C}_{4} \mathrm{AF}$ \\
\hline \hline \multirow{2}{*}{$\mathrm{A}$} & \multirow{2}{*}{ BIST } & 57.5 & 19.6 & 9.4 & 9.3 \\
\cline { 3 - 6 } & & 60.8 & 18.9 & 5.8 & 12.1 \\
\cline { 3 - 6 } & Certified & 66.1 & 17.9 & 5.0 & 11.1 \\
\hline \hline
\end{tabular}

Table 1 (b) Chemical composition of clinkers (unit : \%)

\begin{tabular}{c|c|c|c|c|c|c|c|c|c|c|c|c}
\hline Clinker & $\mathrm{SiO}_{2}$ & $\mathrm{Al}_{2} \mathrm{O}_{3}$ & $\mathrm{Fe}_{2} \mathrm{O}_{3}$ & $\mathrm{CaO}$ & $\mathrm{MgO}$ & $\mathrm{SO}_{3}$ & $\mathrm{Na}_{2} \mathrm{O}$ & $\mathrm{K}_{2} \mathrm{O}$ & $\mathrm{TiO}_{2}$ & $\mathrm{P}_{2} \mathrm{O}_{5}$ & $\mathrm{MnO}$ & $\mathrm{f} . \mathrm{CaO}$ \\
\hline \hline $\mathrm{A}$ & 21.97 & 5.48 & 3.04 & 65.82 & 1.27 & 0.81 & 0.31 & 0.36 & 0.30 & 0.30 & 0.04 & 0.30 \\
\hline NIST & 22.57 & 4.74 & 3.98 & 66.49 & 0.90 & 0.27 & 0.10 & 0.35 & 0.24 & 0.07 & 0.01 & 0.14 \\
\hline
\end{tabular}

as they offer easy handling and provide a representative sample. However, how the preparation conditions for the sample influence the calculated value and what the optimum conditions for applying powder X-ray diffraction and the Rietveld method to clinker and cement have not been adequately analyzed and argued.

We therefore examined the influence of the fineness of the clinkers. We will suggest appropriate preparation conditions for a calculated composition using the Rietveld method.

\section{EXPERIMENTAL}

\section{1 Materials}

Table 1 shows the chemical and mineral composition of the portoland cement clinkers used, as determined by JIS R 5204 : 2002, “Chemical Analysis Method of Cement by X-ray Fluorescence," and Japan Cement Association Standard (JCAS) I-01 : 1997, "Determination Method of Free Calcium Oxide." All of the clinker was manufactured in commercial plants. NIST clinker SRM-2688 was used as a reference material. Table 1 also provides certified values for SRM 2688 expressed as mass fractions.

\section{2 Sample Preparation}

The clinker samples were crushed with a jaw crusher to a particle size of less than $3.4 \mathrm{~mm}$. Samples for $\mathrm{X}$-ray powder diffraction were produced in a tungsten carbide disc mill or planetary ball mill equipped with stainless-steel vials and balls for dry processing. Clinkers of different fineness were obtained by adjusting the milling time and the grinding agent used, specifically diethylene glycol (DEG). The amount of grinding aids was about $0.05 \%$ of the clinker weight being ground. The fineness of the sample was measured according to JIS R 5201 : 1997, "Physical Testing Methods for Cement."

\section{3 Powdery X-ray diffraction and the Rietveld method}

X-Ray Diffraction data were collected using a Bruker-AXS D8 Advance employing $\mathrm{CuK} \alpha$ radiation $(\lambda=$ $0.154 \mathrm{~nm})$. The samples were scanned at between 10 and 65deg with a high-speed detector. The step size was $0.019 \mathrm{deg} / \mathrm{step}$. The $\mathrm{X}$-ray tube power was $40 \mathrm{kV}$ at $40 \mathrm{~mA}$.

A Rietveld calculation for the clinker mineral composition was performed using TOPAS version 3 from Bruker-AXS. This software uses a fundamental parameter method for refinement. In the analysis of the clinker, the phases of alite (M3 polymorphs), belite (beta polymorphs), aluminate (cubic and orthorhombic polymorphs) and ferrite were quantified. The crystal structure models were assumed to be basically similar to those in the reference literature. ${ }^{4)}$ The MarchDollase function was used for alite in order to correct for the preferred orientation. The correction of the height of the sample surface refined by the Rietveld method required adjustment of $80 \mu \mathrm{m}$ or less. For all of the samples measured in this study, the goodness-offit indicator S (=Rwp/Rexp) ranged from 1.5 to 1.9 ; these values were evaluated as excellent. Only about five minutes were required to obtain accurate XRD data and to measure the mineral composition by the Rietveld method.

\section{4 Point-count method for mineral}

A point-counting procedure using optical microscopy confirmed the mineral composition. The polished section of the clinker was etched using an acetic acid vapor, and then counted at least 2000 points.

\section{Results and Discussion}

\section{1 The Influence of the sample fineness}

Fig. 1 illustrates the relationship between the Blaine specific surface area (Blaine value) of the ground clin- 

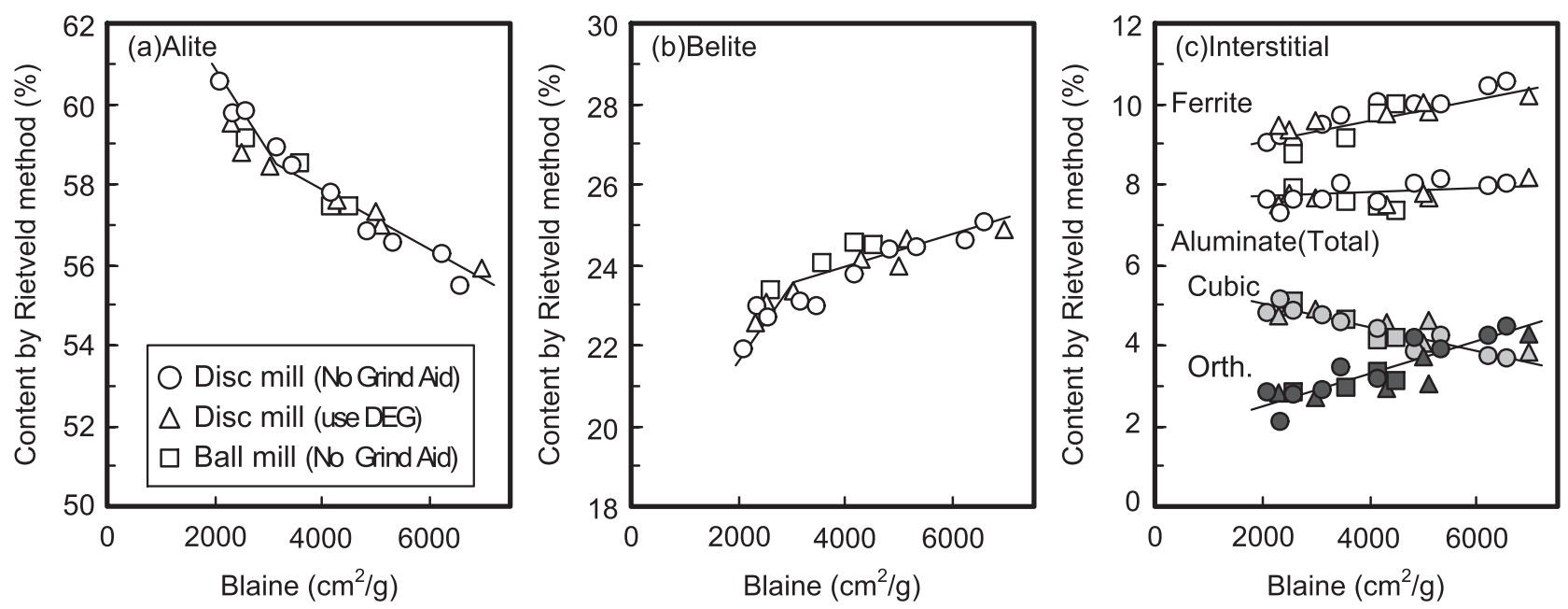

Fig. 1 Effect of the Blaine value of the clinker on the mineral composition calculated by the Rietveld method (using clinker $\mathrm{A}$ )

Table 2 Average change in each mineral when the Blaine value increases $1000 \mathrm{~cm}^{2} / \mathrm{g}$

\begin{tabular}{|c|c|c|}
\hline \multicolumn{2}{|l|}{ Mineral } & Change in content (\%) \\
\hline \multicolumn{2}{|l|}{ Alite } & $-0.9\left(-1.9^{*}\right)$ \\
\hline \multicolumn{2}{|l|}{ Belite } & $+0.5\left(+1.1^{*}\right)$ \\
\hline \multirow[t]{2}{*}{ Aluminate } & Cubic & -0.3 \\
\hline & Ortho. & +0.4 \\
\hline \multicolumn{2}{|l|}{ Ferrite } & +0.3 \\
\hline
\end{tabular}

*Blaine value is $3000 \mathrm{~cm}^{2} / \mathrm{g}$ or less.

ker and mineral composition as determined by the Rietveld method. The calculated amount of alite and cubic aluminate decreases when the Blaine value of the sample increases. In contrast, the calculated amounts of belite, orthorhombic aluminate and ferrite increase with the Blaine value.

The effect of the Blaine value on the calculated content of alite and belite were large when the Blaine value was less than $3000 \mathrm{~cm}^{2} / \mathrm{g}$. Table 2 shows the average change in each mineral when the Blaine value of the sample increases by $1000 \mathrm{~cm}^{2} / \mathrm{g}$.

It was found that this tendency is not affected by the different types of mills, such as ball mills or disc mills. Moreover, the grinding aid used has no influence on the qualification and tendency of change in each phase. This quantitative aspect agrees with that of Enders et al., ${ }^{5)}$ indicating no significant influence of the grinding aid used on the quality of the analysis. In sample preparation for clinker with the Rietveld method, the above results indicate that the major factor is the Blaine value of the sample rather than the type of grinding mill or grinding aid used.

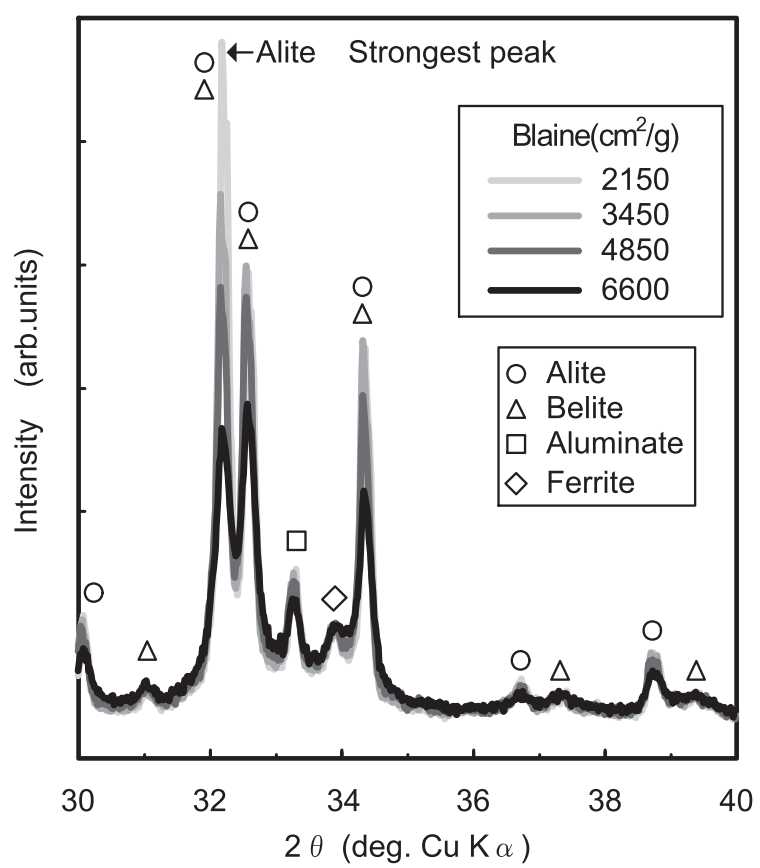

Fig. 2 Observed X-ray diffraction pattern for various sample finenesses

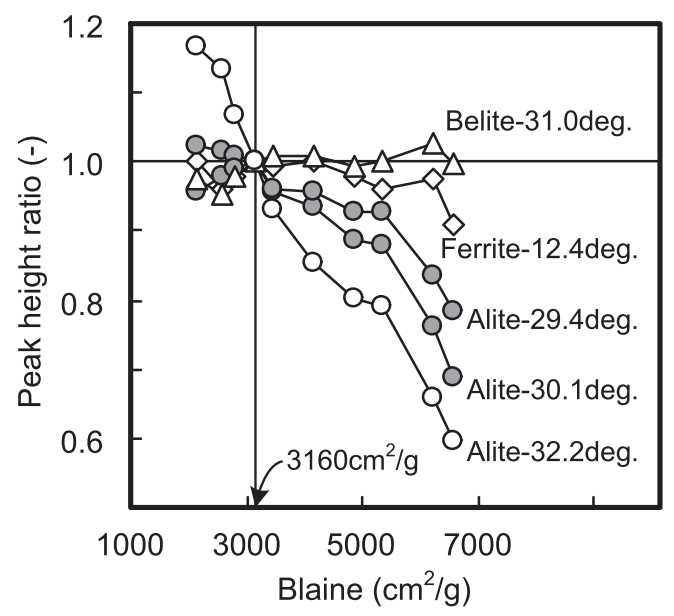

Fig. 3 Effect of Blaine value on the peak height ratio 


\section{2 Cause of Variation in Mineral Content}

In the Rietveld method, the literature has already discussed $^{5,6,7)}$ that the content of alite decreases with grinding time or grinding energy. The literature describes belite as increasing in a complementary manner because most peaks of belite overlap those of alite. In this study, we assumed the following reasons for the change with fineness of the clinker samples,

(1) Preferred orientation of alite.

(2) Loss of crystalline nature of alite.

Fig. 2 depicts the collected XRD profiles of the clinker under measurement conditions in which only the Blaine value of the sample is different. Fig. 3 outlines the observed peak-height ratios based on a Blaine value of $3160 \mathrm{~cm}^{2} / \mathrm{g}$. With increasing Blaine values of the sample, the strongest alite diffraction peak, the $32.2 \mathrm{deg}$ peak, decreases preferentially due to an increase in a particular preferred orientation. In general, minerals with specific shapes like needles or plates tend to become oriented during sample preparation. Alite crystal has a plate form. The non-random arrangement of crystal in the pressed pellet modifies the diffraction pattern, with a loss of certain orientations and relative changes in peak intensities occurring at the same time. An effective correction of the preferred orientation using the March-Dollase function was expected but when the Blaine value was $3000 \mathrm{~cm}^{2} / \mathrm{g}$ or less, this function for alite crystal was typically unable to effect a correction, and the calculated estimate of alite was thus too large. We assumed that the overlapping of the belite and alite peaks is the reason for the complementary increasing in belite content with fineness. On the other hand, the fast decrease in calculated alite content tended to be slower at the Blaine values greater than $3000 \mathrm{~cm}^{2} / \mathrm{g}$. This observation indicates that the amounts of preferred orientation decreases with sample fineness and/or that the correction of the preferred orientation by the March-Dollase function works properly.

However, the calculated alite content continued decreasing at Blaine values greater than $3000 \mathrm{~cm}^{2} / \mathrm{g}$. An inspection of the diffraction pattern reveals that the intensity of all alite peaks decreases with the Blaine value (Figs 2, 3). The width of the peaks increases at the same time. The observation of both decreasing intensity and increasing peak width indicates that the crystallite was smaller.

The literature has already reported ${ }^{8)}$ that overgrinding is associated with the lower crystal structure of alite. In this study the observed change in alite content might be caused not only by the smaller crystal size but also by increasing strain or defects in the crystal structure with grinding time or grinding energy.

Decreasing crystal size or the development of lattice strain in the grinding process might also be closely linked to the grindability of minerals. From this standpoint, we could demonstrate that the grindability of alite was better than that of belite and ferrite. This quantitative aspect agrees with that of Hell et al. ${ }^{9)}$ who indicated that the belite and ferrite are harder to grind than alite.

In addition, it was noted that the same properties apply to cubic aluminate, whose content decreases with the Blaine value. Still, the total content of aluminate was largely unaffected by the fineness of the sample. The aluminate forms could be recognized based on the XRD pattern, mainly related to the $33.5 \mathrm{deg}$ peak, which is a singlet for cubic aluminate and a doublet for orthorhombic aluminate. ${ }^{10)}$ With increased grinding, the crystal size of the cubic aluminate and the singlet peak height decrease and the pattern can be recognized as a doublet peak of orthorhombic aluminate.
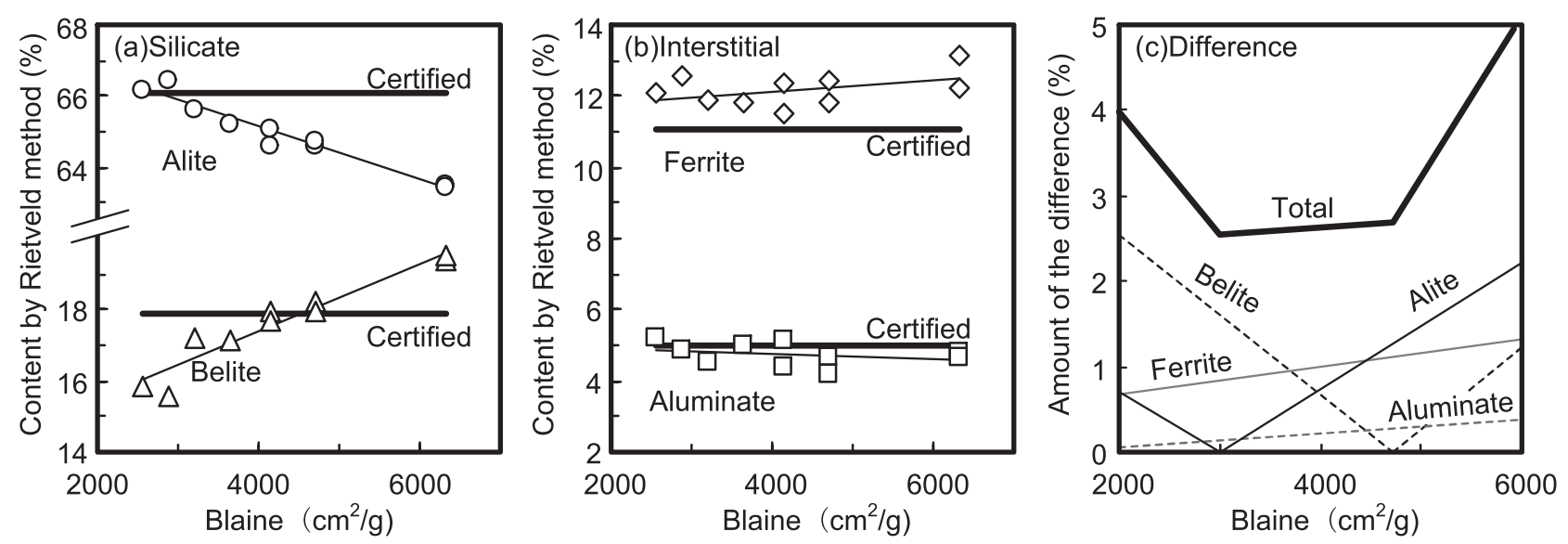

Fig. 4 Effect of the Blaine value of clinker on the calculated content of clinker minerals (NIST clinker) 
Table 3 Mineral composition of the clinkers, calculated using the Rietveld method and Point-count method

\begin{tabular}{l|l|c|c}
\hline \multirow{2}{*}{ Sample } & \multirow{2}{*}{ Mineral } & \multicolumn{2}{|c}{ Content (\%) } \\
\cline { 3 - 4 } & & Rietveld method & Point-Count method* \\
\hline \hline \multirow{2}{*}{$\begin{array}{l}\text { Clinker : NIST } \\
\text { Blaine : } 3200 \mathrm{~cm}^{2} / \mathrm{g}\end{array}$} & Alite & 65.6 & 64.4 \\
\cline { 2 - 4 } & Belite & 17.2 & 17.5 \\
\cline { 2 - 4 } & Interstitial & 16.4 & 18.0 \\
\hline \hline \multirow{2}{*}{$\begin{array}{l}\text { Clinker : A } \\
\text { Blaine : } 3510 \mathrm{~cm}^{2} / \mathrm{g}\end{array}$} & Alite & 58.9 & 60.3 \\
\cline { 2 - 4 } & Belite & 23.1 & 22.1 \\
\cline { 2 - 4 } & Interstitial & 17.1 & 17.6 \\
\hline
\end{tabular}

${ }^{*}$ Convert vol\% to mass \% by reference to ASTM C $1356: 2007$

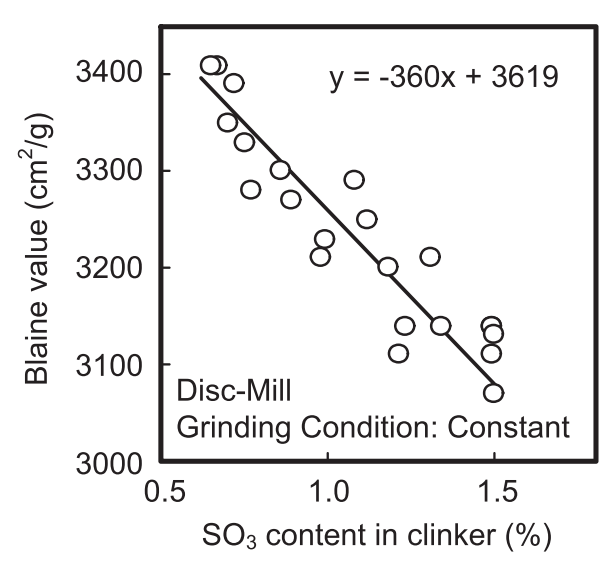

Fig. 5 Relationship between $\mathrm{SO}_{3}$ content in clinker and the Blaine value for the same grinding conditions

\section{3 Appropriate fineness for Rietveld calculations}

We studied the appropriate fineness of clinker samples to accurately measure the mineral composition, using NIST clinker whose mineral composition was certified. The fineness of the NIST clinker was adjusted by a planet ball mill. Fig. 4 plots the relationship between the Blaine value of the sample and the mineral composition by the Rietveld method. The influence of the Blaine value of the sample on the calculated mineral composition was reasonably consistent with the relation seen in Table 2. Unfortunately, the calculated contents of all the clinker minerals did not completely match the certified values at various Blaine values. The appropriate Blaine value was determined to minimize the difference between the content measured by the Rietveld method and certified value. Fig. 4(c) depicts the relation between the Blaine value and the difference in the values as compared with the calculated content and the certified value in each mineral. The total difference between the content of each mineral and the certified value was assumed to be minimized (to about $2.5 \%$ ) by making the Blaine value of clinker fall within the range of 3000 to $4500 \mathrm{~cm}^{2} / \mathrm{g}$.
We ran tests to compare the mineral composition as measured by the Rietveld method and the Pointcount method. Table 3 presents the result of the confirmation. The mineral compositions calculated by the Rietveld method at an appropriate sample fineness and the point-count procedure were consistent within the point-count deviation. ${ }^{11}$

\section{4 Influence of clinker grindability}

In actual quality control in a cement plant, clinker samples transported to the laboratory were crushed with a jaw crusher to a particle size less than $5 \mathrm{~mm}$. The samples used for XRD analysis were produced with a grinding apparatus such as disc mill or ball mill, under constant conditions. As mentioned above, the grinding conditions such as the grinding time, sample feed, etc., need to be adjusted so that the sample fineness remains as constant as possible between 3000 and $4500 \mathrm{~cm}^{2} / \mathrm{g}$. However, for some clinker samples, even careful preparation does not always produce the same sample fineness because the grindability of the clinkers is not always the same. Hence, we tested the influence of the $\mathrm{SO}_{3}$ in clinker on the Blaine value under constant grinding conditions and calculated the mineral composition using the Rietveld method. This test is based on the idea that $\mathrm{SO}_{3}$ content in the clinker causes low grindability. ${ }^{12)}$

Fig. 5 illustrates the relation between the $\mathrm{SO}_{3}$ content in clinker and the Blaine value of clinker produced under the same grinding conditions. It is to be anticipated that the Blaine value of a sample under these grinding conditions will decrease by $360 \mathrm{~cm}^{2} / \mathrm{g}$ with an increase in $\mathrm{SO}_{3}$ content from 0.5 to $1.5 \%$. This variation in the Blaine value is small enough that little change was detected for the calculated content (Table 2). Consequently, under the same grinding conditions, the mineral composition calculated by the Rietveld method is not affected by the grindability of the clinker if the $\mathrm{SO}_{3}$ content in the clinker is within 0.5 to $1.5 \%$, which covers most industrial clinker. 


\section{5 Preparation for Measurement of Cement Sample}

Sample preparation might affect not only the clinker but also the cement. The influence of fineness on the calculated mineral composition of cement was predicted to be similar to that of clinker. However, the Blaine value of portland cement manufactured domestically or internationally is between 3000 and $4500 \mathrm{~cm}^{2} / \mathrm{g}$ except for some special kind of cement. Moreover, grinding the cement sample in preparation for measurement causes crucial changes in the gypsum content calculated by the Rietveld method. ${ }^{5)}$ At present, this confirms that no preparatory grinding procedure is necessary for the measuring cement by the Rietveld method.

\section{CONCLUSION}

We investigated the appropriate sample preparation conditions of clinker and cement for X-ray diffraction and the Rietveld method with an automated laboratory system while keeping an actual cement plant in mind. The following results were obtained.

(1) The fineness of clinker sample affects the calculated mineral composition especially alite and belite, as determined by the Rietveld method. It is appropriate to maintain the Blaine specific surface area constant within a range of 3000 to $4500 \mathrm{~cm}^{2} / \mathrm{g}$ to achieve the calculated clinker mineral composition.

(2) It is believed that the mineral composition calculated by the Rietveld method is not affected by the grindability of clinker or the use of grinding aid. In addition, it appears that no grind preparation is necessary for cement measurement.

In the near future quantitative $\mathrm{X}$-ray diffraction of clinker and cement will become a more feasible tool for automated laboratory systems in cement plants. This paper demonstrates that appropriate mineral composition can be obtained using the Rietveld method by adjusting the grind conditions with the Blaine value of the clinker as describe above.

\section{REFERENCES :}

1) I. Odler : "Hydration, Setting and Hardening of Portland Cement," Edited by P. C. Hewlett : "Lea's Chemistry of Cement and Concrete, Fourth Edition," pp. 241-297, John Wiley \& Sons Inc. New
York, US (1998)

2) R. Schmidt and A. Kern : "Quantitative XRD Phase Analysis,” World Cement, Vol. 32, No. 2, pp. 35-42 (2001)

3) M. Enders : "Quantitative Phase Analysis," World Cement, Vol. 38, No. 6, pp. 45-50 (2007)

4) P. Stutzman and S. Leight: "NIST Technical Note 1441-Phase Composition Analysis of the NIST Reference Clinkers by Optical Microscopy and X-ray Powder Diffraction," pp. 34-43 (2002)

5) M. Enders : "Quantitative XRD-Analysis in Automated Cement Laboratories : Requirements for the Sample Preparation," ZKG international, Vol. 56, No. 5, pp. 54-62 (2003)

6) M. Enders : "Sample Preparation for Quantitative $\mathrm{X}$-ray Diffraction in Cement Plants: Sources of Errors and Solutions," ZKG international, Vol. 58, No. 4, pp. 28-37 (2005)

7) L. D. Mitchell, P. S. Whitfield, J. J. Beaudoin : "The Effects of Particle Statistics on Quantitative Rietveld Analysis of Cement," Proceeding of the 12th International Congress on the Chemistry of Cement, Montreal, Paper No. TH1-08.3 (2007)

8) K. Mori et al. : "Structual and Hydration Properties of Amorphous Tricalcium Silicate," Cement and Concrete Research, Vol. 36, pp. 2033-2038 (2006)

9) L. M. Hills : "The Effect of Clinker Microstructure on Grindability : Literature Review Database," Research and Development Information, RP331, Portland Cement Association, Skokie, US (1995)

10) L. Gobbo, L. Sant'Agostino, L. Garcez : " $\mathrm{C}_{3} A$ polymorphs related to industrial clinker alkalies content," Cement and Concrete Research, Vol. 34 , pp. 657-664 (2004)

11) Report of the technical committee on cement chemistry C-12 : "Study on the comparison between measured amount obtained by independent determination techniques for clinker constituents," Japan cement association, p. 243 (2008)

12) K. Shimosaka et al. : "Influence of sulfur oxide in clinker on the properties of clinker and cement," Cement Science and Concrete technology, Vol.56, pp. 9-16 (2002) 